\title{
A Static Model for Electrolyte-Gated Organic Field-Effect Transistors
}

\author{
Deyu Tu, Lars Herlogsson, Loig Kergoat, Xavier Crispin and Magnus Berggren
}

\section{Linköping University Post Print}

N.B.: When citing this work, cite the original article.

(C2009 IEEE. Personal use of this material is permitted. However, permission to reprint/republish this material for advertising or promotional purposes or for creating new collective works for resale or redistribution to servers or lists, or to reuse any copyrighted component of this work in other works must be obtained from the IEEE.

Deyu Tu, Lars Herlogsson, Loig Kergoat, Xavier Crispin and Magnus Berggren, A Static Model for Electrolyte-Gated Organic Field-Effect Transistors, 2011, IEEE Transactions on Electron Devices, (58), 10, 3574-3582.

http://dx.doi.org/10.1109/TED.2011.2162648

Postprint available at: Linköping University Electronic Press

http://urn.kb.se/resolve?urn=urn:nbn:se:liu:diva-71549 


\title{
A static model for electrolyte-gated organic field effect transistors
}

\author{
Deyu Tu, Lars Herlogsson, Loïg Kergoat, Xavier Crispin, Magnus Berggren, and Robert Forchheimer
}

\begin{abstract}
We present a DC model to simulate the static performance of electrolyte-gated organic field effect transistors. The channel current is expressed as charge drift transport under electric field. The charges accumulated in the channel are considered being contributed from voltage-dependent electric double layer capacitance. The voltage dependent contact effect and short channel effect are also taken into account in this model. A straightforward and efficient methodology is presented to extract the model parameters. The versatility of this model is discussed as well. The model is verified by the good agreement between simulation and experimental data.
\end{abstract}

Index Terms - static model, electric double layer capacitance, field effect transistors, parameter extraction, polymer electrolyte

\section{INTRODUCTION}

$\mathrm{P}$ OLYMER semiconductors have attracted highly notable attention and been widely studied for their potential applications in electronic devices and circuits, since the discovery of electrical conduction in plastics [1]. Towards flexible, low-cost, and large-area integrated circuits, polymer transistors have been known as an ideal candidate with their unique features, such as printability and tunable functionality [2]. Among all various transistors based on polymer materials, field effect transistors are the most promising and deeply explored, due to their similarity with traditional silicon thin-film transistors [3]. However, it has been quite challenging to make polymer or organic field effect transistors work at low voltage with conventional silicon dioxide [4] or polymer insulator [5] as gate dielectric. Therefore, tremendous effort has been devoted to the engineered of gate dielectrics, in order to lower the control voltage for field effect transistors [6 10]. The

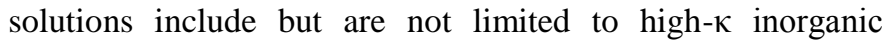
materials [6,7], self-assembled molecular monolayer [8], and ion-gel gate $[9,10]$. Particularly, the devices with ion-gel gate dielectric have an excellent turn-on conductance at low operating voltage $(<2 \mathrm{~V})$, but suffer from extremely slow switching speed (approximately in seconds) [10], which is

Manuscript received April 21, 2011. This work was supported by "OPEN" project at the Center of Organic Electronics (COE) at Linköping University, Sweden, funded by the Strategic Research Foundation SSF.

Deyu Tu and Robert Forchheimer are with the Information Coding, ISY Linköping University, SE-581 83 Linköping Sweden (e-mail: robert@ isy.liu.se).

Lars Herlogsson, Loïg Kergoat, Xavier Crispin, and Magnus Berggren are with the Organic Electronics, ITN Linköping University, SE-601 74 Norrköping Sweden. comparable with_electrochemical transistors [11, 12].

Recently, a polymer electrolyte (copolymer of vinyl phosphonic acid and acrylic acid, P(VPA-AA)) has been demonstrated as a gate dielectric in polymer field effect transistors, achieving an operating voltage less than $1 \mathrm{~V}$ [13 15]. With the capability to form electric double layer capacitors (EDLCs), P(VPA-AA) indicates a new generation of organic field effect transistors (OFETs), named electrolyte-gated organic field effect transistors (EGOFETs) or EDLC-OFETs. The formation of EDLCs is a complex electrochemical process, which results nonlinear and voltage-dependent capacitance, and pretty crucial to device's performance. So far, no device model, taking this effect into account, has been published.

Here, we propose a model to describe the static electrical behaviors of EGOFETs. The model is based on charge drift in presence of accumulated charges in the channel, covering subthreshold, linear and saturation regimes of the EGOFETs. The charges accumulated are contributed from the field effect caused by electric double layer capacitance dependent voltage biased. Dynamic equilibrium of the electrochemical process in EDLCs is discussed to interpret the nonlinearity and voltage dependence of the capacitance. Contact barrier dependent on gate voltage and short channel effect are taken into account in this model. The extraction of physical parameters from experimental results is demonstrated. With the extracted parameters, good agreements have been found between model simulation and experimental data.

\section{II.EDLCS AND EGOFETS}

With huge capacitance and high charging rate, EDLCs, also known as supercapacitors, have been widely investigated as energy-storage devices [16]. The extraordinary capacitance per unit area of EDLCs is up to $500 \mu \mathrm{F} / \mathrm{cm}^{2}$ [17], over three orders higher in magnitude than that of 100-nm-thick hafnium oxide $\left(\kappa=25,0.22 \mu \mathrm{F} / \mathrm{cm}^{2}\right)$ [18], which is the best known high- $\kappa$ dielectric applied in traditional silicon-based metal-oxide-semiconductor field effect transistors. This implies that EDLC is an efficient solution to lower the operating voltages for organic or polymer field effect transistors. Driven by potential bias, the anions/cations in the electrolyte are attracted to the charged electrode, forming an electric double layer, composed of a Helmholtz layer and a diffuse layer, schematically shown in Fig. 1(a). In this paper, the charge contribution to transport in the channel of the Helmholtz layer is considered as a non-neglectable part. Specifically, the 
polymer electrolyte referred to here is a random copolymer, $\mathrm{P}(\mathrm{VPA}-\mathrm{AA})$, with the chemical structure as presented in Fig. 1(b). The protons in P(VPA-AA) are mobile ions, capable of travelling under electric field or diffusion. A top-gate bottom-contact configuration of field effect transistors was chosen for the discussed EGOFETs [13], sketched in Fig. 1(c). In the initial state of polymer electrolyte gate dielectric, we assume that the protons are uniformly distributed in the polymer matrix. When a negative voltage is applied to the gate, the protons are pulled to the gate, causing a concentration gradient of ions, which drives protons away from the gate. When these two opposite movements of protons in the polymer gate dielectric reach equilibrium, a layer of protons close to the gate contact is established. Since polymer chains as anions are considered to be immobile, the negative charges are accumulated at the interface between the gate electrolyte and semiconductor. This charge contribution of the electric double layer capacitor is taken into account in this model, although there is probably a part of ions penetrating into the bulk

(a)

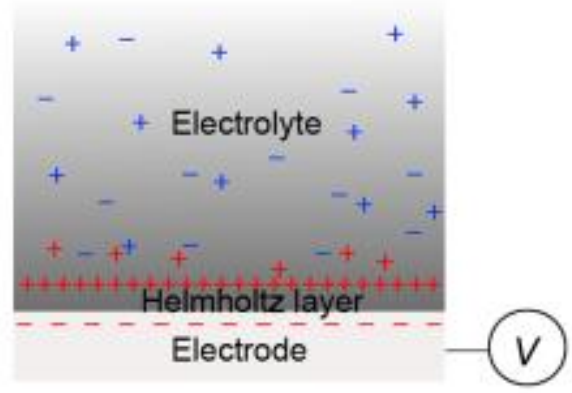

(b)

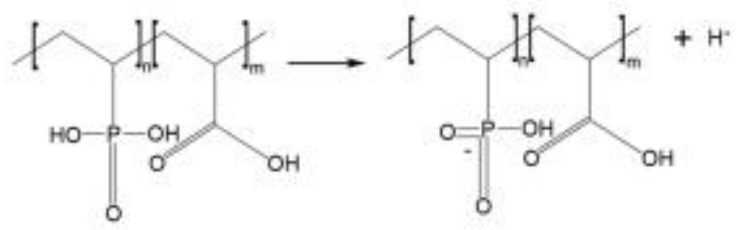

(c)

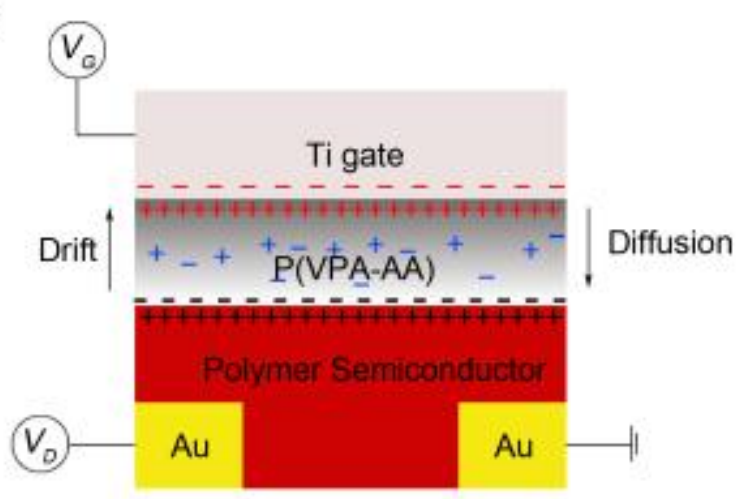

Fig. 1. (a) Schematic of electric double layer at electrolyte and electrode interface, when the electrode is applied a potential. (b) The molecular structure of P(VPA-AA) and its deprotonated process under a negative bias. (c) The sketched device structure of EGOFETs discussed in this work. The arrows indicate directions of proton movements driven by electric field or diffusion. polymer semiconductor layer [13]. The undesired ion doping leads to very slow switching speed and huge hysteresis, making EGOFETs like electrochemical transistors. At certain voltage bias, EGOFETs can be operated only in field effect mode. Therefore, the ion penetration into semiconductor channel is neglected in this work.

\section{MODEL THEORY AND DERIVATION}

Despite that many theories are proposed and reported to describe behaviors of various organic field effect transistors, the widely known ones are based on charge drift [19] in presence of tail-distributed traps [20] and variable range hopping [21]. For the charge drift model, a critical point is to figure out the quantity of charges at the dielectric-semiconductor interface. In our model, the charge contribution is considered to be provided by electric double layer capacitance, which is nonlinear and voltage dependent. Moreover, a very important common point among different theories is the voltage dependent mobility, which has to be taken into account. Here, we consider a few common points in the modeling of organic field effect transistors, including charge drift transport, voltage dependent mobility, contact effect and gate leakage current, as well as the contribution of EDLCs to propose an EGOFET-exclusive compact model. The detailed derivation is shown below. To simplify the derivation, all equations are given for the n-type field effect transistors first. In our case, the P(VPA-AA)-gated transistors are p-type and polarity of voltages and currents will be reversed.

\section{A. Electric double layer capacitance}

The electric double layer is created at the interfaces between electrolyte and metal gate and between the electrolyte and the/semiconductor. Since the dimension of a proton is much smaller than the dimension of the anionic group in $\mathrm{P}$ (VPA-AA), the overall capacitance is actually limited by the smallest electric double layer located at the polyelectrolyte -semiconductor interface. At this interface, the electrolyte is divided into two zones: a compact layer constituted of the first monolayer of polyanions in contact with the semiconductor and, and a diffusion layer composed of polyelectrolyte chains partially depleted from the protons. The compact layer is modeled by Helmholtz [22], giving a capacitance dependent on the distance between the metal surface and a plane of ions. In the diffusion layer, the two motions of mobile protons, drift by electrical field and diffusion by concentration gradient, reach equilibrium at steady state. This complicated process is described by the Gouy-Chapman theory, which was revised by Stern later, yielding a correlation between surface charges and electric surface potential to determinate the nonlinear capacitance [23]. Interestingly, for low ion concentrations, the capacitance is expected to change abruptly around the potential of zero charge [24], while for high concentration of ions, as it is the case in the polyelectrolyte used for the transistor, the ideal model leads to a constant capacitance versus voltage. From theory, the capacitance versus voltage in the polyelectrolyte used for the transistor is expected to be small. Actual electric double layers however, always display a smooth evolution of the capacitance versus voltage at high ion concentration [25]. 

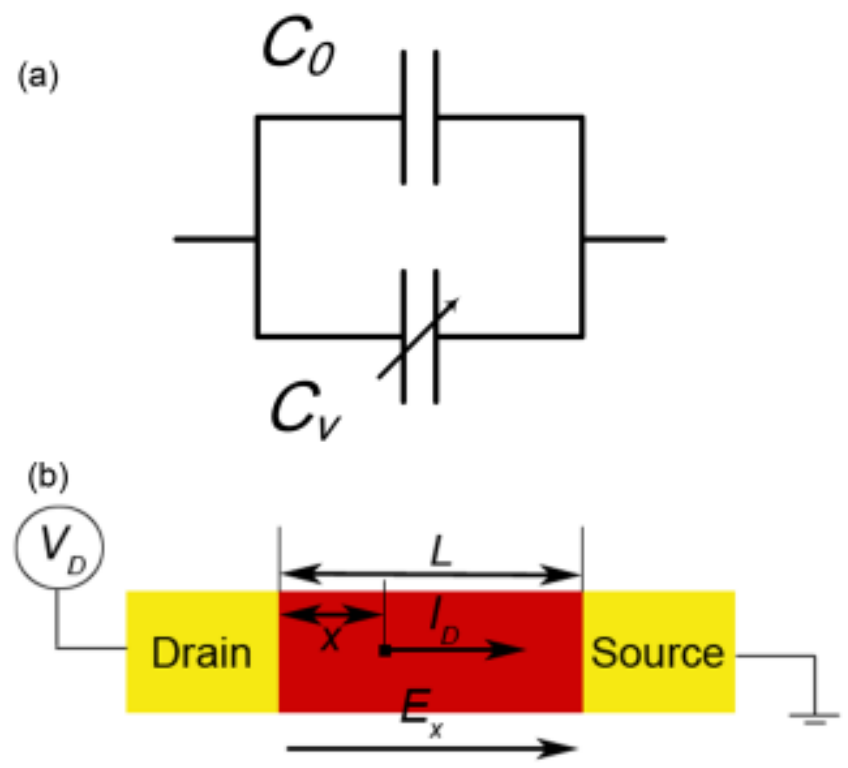

(c)

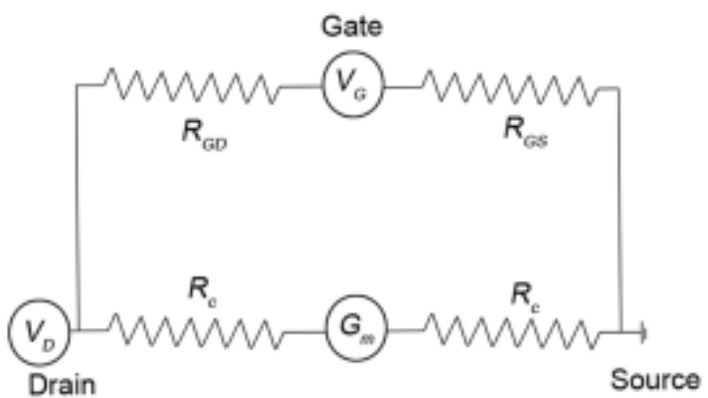

Fig. 2. (a) The circuit diagram of revised Two Branches Model of EDLCs. Voltage-dependent capacitor $C_{v}$ and voltage-independent capacitor $C_{0}$ are connected in series. (b) Schematic of transistor channel, the accumulated charges in channel drift under electrical field, forming the channel current. (c) A static equivalent circuit of EGOFETs, parasitic capacitance is neglected. The $G_{m}$ is gate voltage-dependent channel conductivity, and $R_{G D}$ or $R_{G S}$ is the negligible resistance between gate and drain or source, respectively.

Such deviation from ideal models is here included in the following model. We consider this voltage-dependent capacitance summed of two parts, one is proportional to a certain power of voltage but the other is constant, for this model. The equivalent circuit of the revised two branches model for electric double layer capacitor is presented in Fig. 2(a). So, the capacitance of electric double layer $C(V)$, at a certain position $x$ in the channel is given by $C(V)=C_{0}+C_{v}\left(V_{G T}-V(x)\right)^{x} \quad(0<x<L, \quad \chi>0)$, where $C_{0}$ is the voltage-independent of capacitance, $C_{v}$ is the voltage-dependent capacitance, $V_{G T}$ is the gate voltage $V_{G}$ minus the threshold voltage $V_{T}, V(x)$ is the potential at position $x$ between source and drain, $\chi$ is the EDLC voltage-dependent factor, and $L$ is the channel length.

\section{B. Charge drift model}

To describe the DC behavior of charge transport in the device channel, a widely-accepted charge drift model [19] is presented here. As shown in Fig. 2(b), the channel current at point $x$ per unit width $\left(I_{D} / W\right)$ is given by $I_{D} / W=\mu(x) Q(x)|E(x)|$, and $\mu(x)$ is the voltage-dependent charge mobility, $Q(x)$ is the accumulated charges and $E(x)$ is the electric field given by the drain voltage along the channel, at point $x$ in the channel. The current is considered to be consistent along the channel.

From the theories of organic field effect transistors [20, 21], the mobility $\mu(x)$ is related to the voltage potential at that location and can be written as $\mu(x)=\mu_{0}\left(V_{G T^{-}} V(x)\right)^{\gamma}(\gamma>0)$, where $\mu_{0}$ is the low-field mobility and $\gamma$ is the mobility enhancement factor.

With this capacitance of EDLCs, the charge accumulated at the position $x$ in the channel is rewritten as

$$
\begin{aligned}
Q(x) & =C(V)\left(V_{G T}-V(x)\right) \\
& =C_{0}\left(V_{G T}-V(x)\right)+C_{i}\left(V_{G T}-V(x)\right)^{\chi+1} .
\end{aligned}
$$

In addition, $|E(x)|$ is $\partial V(x) / \partial x$.

Here, the channel current can be rewritten as follows,

$$
\begin{gathered}
I_{D}(x) / W=\mu_{0}\left(C_{0}\left(V_{G T}-V(x)\right)^{\gamma+1}\right. \\
\left.+C_{i}\left(V_{G T}-V(x)^{\gamma+\chi+1}\right)\right) \frac{\partial V(x)}{\partial x} .
\end{gathered}
$$

Integrating along channel length $x$, an intermediate equation is given as

$$
\int_{0}^{L}\left(I_{D}(x) / W\right) d x=\int_{0}^{L}\left[\cdots \frac{\partial V(x)}{\partial x}\right] d x=\int_{V_{S}}^{V_{D}}[\cdots] d V(x) .
$$

Usually, the source terminal is grounded in most organic field effect transistor instances and so we assume here. The potential of source $V_{S}$ is set to $0 \mathrm{~V}$ to simplify equation derivation and the current is considered to be consistent along the channel. Therefore, the first channel current formula of EGOFET model is obtained from (3) as

$$
\begin{aligned}
I_{D, \text { lin }} & =\frac{W}{L} \mu_{0}\left[C_{0} \frac{V_{G T}^{\gamma+2}-\left(V_{G T}-V_{D}\right)^{\gamma+2}}{\gamma+2}\right. \\
& \left.+C_{i} \frac{V_{G T}^{\gamma+\chi+2}-\left(V_{G T}-V_{D}\right)^{\gamma+\chi+2}}{\gamma+\chi+2}\right]
\end{aligned}
$$

This equation covers DC behaviors of EGOFETs in linear regime. Furthermore, the saturation regime, subthreshold regime and a few detailed supplements are discussed below to make the model solid and versatile, based on this equation.

\section{Saturation regime}

For saturation regime, the $V_{D}$ is superior to $V_{G T}$, and then the $V_{G T}-V_{D}$ is considered to be zero, approximately. When downscaling the channel length of field effect transistors to less than a few microns, short-channel effects [26] can be observed, which can cause a series of issues to the device characteristics, such as non-saturation, threshold voltage shift, fall of on/off ratio, and so on. Here, a channel length modulation factor $1+\lambda\left(V_{D^{-}} V_{G T}\right)$ is introduced to address a short channel effect in the saturation current equation, where $\lambda$ is the channel length modulation coefficient [27]. Hence, the (4) can be rewritten for the saturation regime in this form 


$$
\begin{aligned}
I_{D, s a t} & =\frac{W}{L} \mu_{0}\left(C_{0} \frac{V_{G T}^{\gamma+2}}{\gamma+2}+C_{i} \frac{V_{G T}^{\gamma+\chi+2}}{\gamma+\chi+2}\right) \\
& \times\left[1+\lambda\left(V_{D}-V_{G T}\right)\right]
\end{aligned}
$$

\section{Subthreshold regime}

Most of the induced charges are trapped in the subthreshold regime, and the charge transport in the channel is not dominated by drift but by diffusion. The charge drift-based model is not accurate to describe device behaviors in the subthreshold regime. The channel current can be presented as in [19], with a similar expression to conventional transistors,

$$
I_{D, \text { sub }}=\frac{W}{L} \mu_{0} C_{0} V_{S S}^{2} \exp \left(\frac{V_{G T}}{V_{S S}}\right)\left[1-\exp \left(\frac{-V_{D}}{V_{S S}}\right)\right],(6)
$$

where $V_{s s}$ is a voltage parameter, reflecting the steepness of the subthreshold characteristics, which can be approximately estimated with the subthreshold slope [28]. The electric double layer capacitance is considered to be negligible, since the gate voltage is rather low in subthreshold regime.

\section{E. Contact effect}

For ideal transistor operation, the contact resistance between source/drain electrodes and semiconductor channel is generally negligible. This contact is named Ohmic contact. However, contact resistances for organic field effect transistors are often quite substantial and play an important role in the charge transport. Fig. 2(c) presents an equivalent circuit of EGOFETs for DC performance without parasitic capacitances. The contact resistances at the source and drain are considered to be symmetric. If the contact resistance $R_{C}$ is considered, the effective drain voltage $V_{D}$, excluded the voltage drop on contact resistance, will be $V_{D}{ }^{\prime}=V_{D^{-}}-2 I_{D} R_{C} / L$.

As reported [29], contact resistance in organic field effect transistors is highly dependent on gate voltage. To take this dependence into account, a power law dependence on $V_{G T}$ is given to the contact resistance in this form $R_{C}(V)=R_{C 0} V_{G T}{ }^{-(\gamma+l)}$ [30], where $R_{C 0}$ is the contact resistance at $V_{G T}=1 \mathrm{~V}$. Here, the contact resistance shares the same factor $\gamma$ as the voltage dependent mobility and this is due to the contact resistance is reciprocal to the amount of accumulated charge carriers times their voltage dependent mobility [30].

\section{F. Threshold voltage shift}

Although electrolyte gate has suppressed short channel effects, we still observe the trend when the channel length shrinks [14]. As referred above, one of short channel effects is the shift of $V_{T}$, which is also called threshold voltage roll-off [31]. This is due to the shrinking channel length lowers the required gate voltage to make the channel conductive, hence the threshold voltage shifts toward the reverse direction of gate voltage switching on the transistor. If the channel length is long enough, the threshold voltage is consistent along the channel length. To address the impact of short channel effect, the shift of $V_{T}$ from the long channel value is reciprocal to the channel length [32], so the $V_{T}$ dependence on channel length is expressed as $V_{T}(L)=V_{T, L}(1-\zeta / L)$, where $\zeta$ is a coefficient, $V_{T, L}$ is the threshold voltage of the long channel transistors, without short channel effect.

The variation of threshold voltage with biasing is another factor which makes the $V_{T}$ shift [33]. The sensitivity of this dependence is expressed as $\delta_{V T}=\partial V_{T} / \partial V_{G}$.

Including this parameter, the threshold voltage can be modified to $V_{T}(L)=V_{T, L}(1-\zeta / L)+V_{G} \delta_{V T}$. In this modification, we have considered the influence of both channel length and voltage bias on threshold voltage.

\section{RESULTS AND DISCUSSION}

To verify the above model, EGOFETs with polymer electrolyte P(VPA-AA) were fabricated and characterized as reported [13]. Two kinds of polymer semiconductors were used respectively, including PTTTT and P3HT. The devices had Au source/drain bottom electrodes with channel length $L=2$ to 50.5 $\mu \mathrm{m}$ and width $W=1000 \mu \mathrm{m}$. The thickness of spin-coated P3HT/PTTTT layer was around $\sim 30 \mathrm{~nm}$ and P(VPA-AA) gate dielectric layer was $100 \mathrm{~nm}$, capped by a Ti gate electrode. The measurements were carried out with a Keithley 4200-SCS semiconductor characterization system in ambient air at room temperature. Here, PTTTT transistors with $L=50.5 / 20.5 / 10.5 / 5.5 / 3.5 / 2 \mu \mathrm{m}$ are presented as example to extract parameters in this model, and then the comparison of
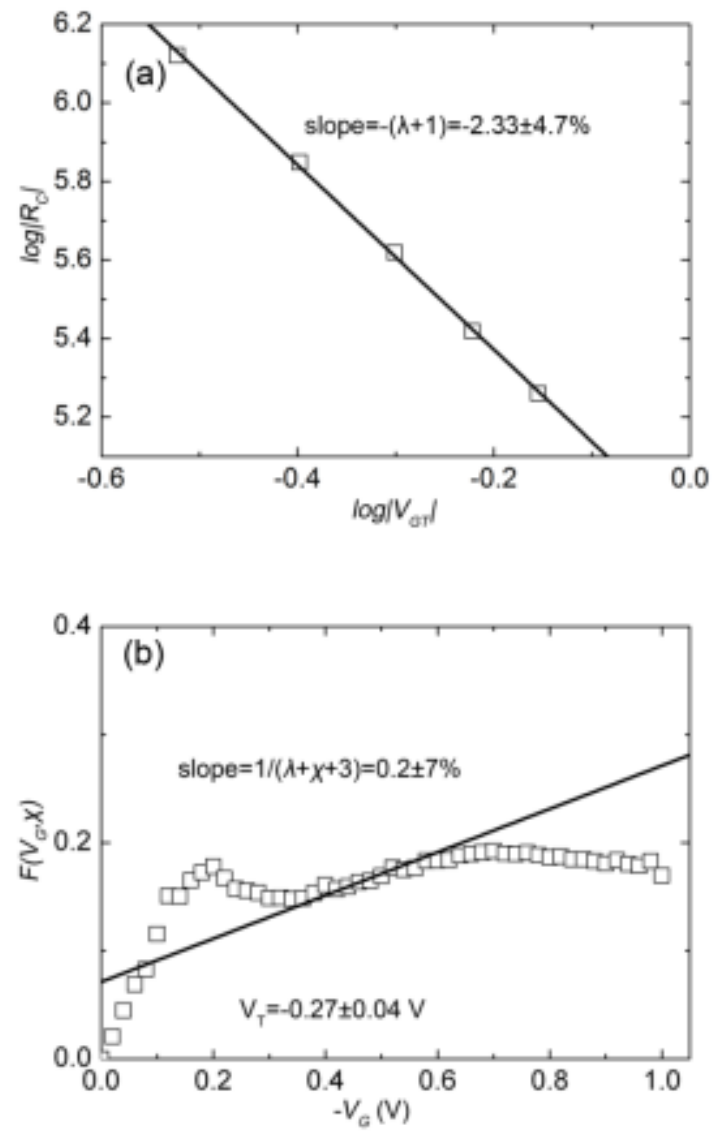

Fig. 3. (a) The contact resistance dependence on voltage is graphed with $\log R_{C}$ versus $\log V_{G T}$. (b) $F\left(V_{G}, \chi\right)$ plot of EGOFET with 50.5- $\mu \mathrm{m}$ channel length. The slope of linear fitting gives the coefficient $\chi$. 
simulation results and experimental data at various channel length is followed in this discussion.

\section{A. Extraction of mobility enhancement factor $\gamma$}

The power law dependence of contact resistance on voltage can be rewritten as $\log R_{C}=\log R_{C 0^{-}}(\gamma+1) \log V_{G T}$.

Unlike the method in [28], this equation provides an alternative way to extract the mobility enhance factor $\gamma$. Here, we extract contact resistance at $V_{G}=0.6 / 0.7 / 0.8 / 0.9 / 1 \mathrm{~V}$ with the method discussed below, respectively. Then the $\gamma$ can be calculated from the relation between $R_{C}$ and $V_{G}$. The $\log R_{C}$ versus $\log V_{G T}$ is plotted in Fig. 3(a) and a linear fitting gives a slope of -2.33 . Hence, we can obtain the mobility enhance factor $\gamma$, which is around 1.33, comparable with those conventional organic field effect transistors [28].

\section{B. Extraction of EDLC factor $\chi$}

To simplify the extraction, we use the saturation equation to obtain the voltage-dependence coefficient $\chi$. In addition, the data are from the transistor with $50.5-\mu \mathrm{m}$ channel length, so the short channel effect is negligible. A function $F\left(V_{G}, \chi\right)$, consisting of $V_{G}$ and $\chi$, is written as

$F\left(V_{G}, \chi\right)=\frac{\int_{V_{T}}^{V_{G}}\left(\frac{1}{\gamma+2} \frac{\partial I_{D}}{\partial V_{G}} V_{G T}-I_{D}\right) d V_{G}}{\frac{1}{\gamma+2} \frac{\partial I_{D}}{\partial V_{G}} V_{G T}-I_{D}}=\frac{V_{G T}}{\gamma+\chi+3} .(7)$

In this equation, $F\left(V_{G}, \chi\right)$ becomes a linear function of gate voltage with only another two coefficients $\gamma$ and $\chi$, and the $\gamma$ is 1.33, as extracted above. From the slope of linear fitting in Fig. 3(b), presenting the plot of the function $F\left(V_{G}, \chi\right)$, the electric double layer capacitance coefficient $\chi$ is calculated to be 0.67 .

\section{Extraction of $L$ modulation coefficient $\lambda$}

From (5), we can see that the increasing current is proportional to the difference of $V_{D}$ and $V_{G T}$ in saturation regime and the slope is $\lambda$. Here, we extract $\lambda$ at fully-on state of transistors, although it is usually related to gate voltage in silicon CMOS transistors [34]. Fig. 4(a) presents the current curves in saturation regime versus $V_{D^{-}} V_{G T}$ and their linear fittings at $V_{G}=-1 \mathrm{~V}$, obtained from transistors with channel

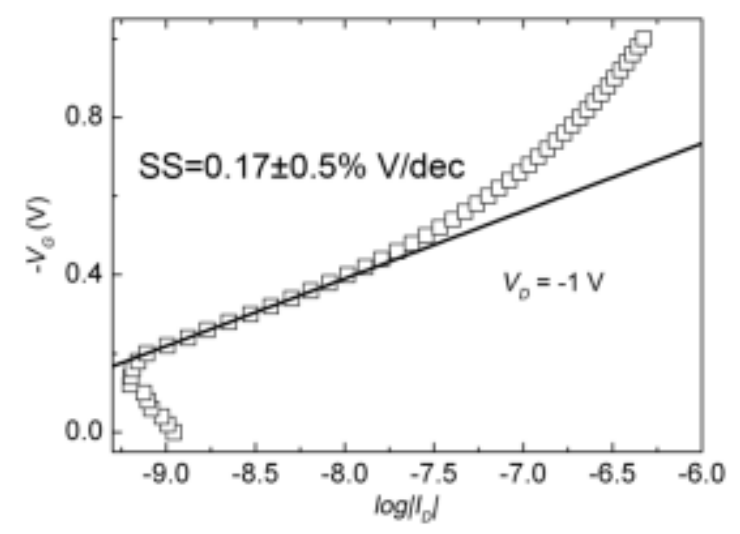

Fig. 5. The subthreshold slope is extracted to estimate the parameter $V_{S S}$.
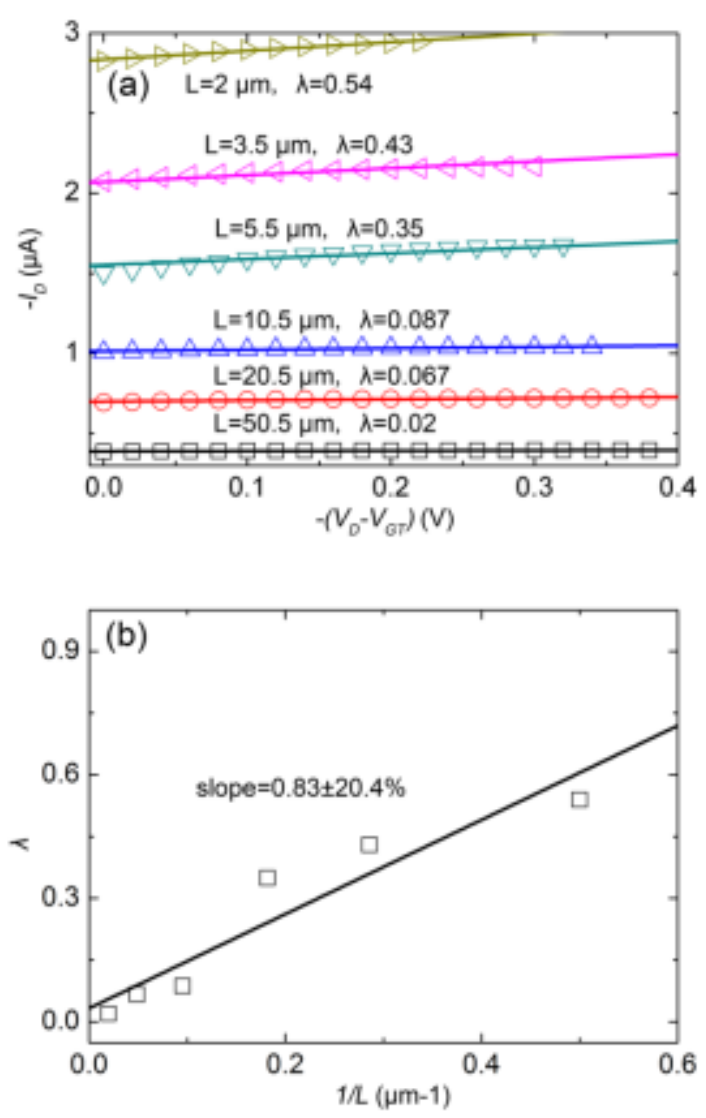

Fig. 4. (a) The output curves of EGOFETs with various channel length in saturation regime at $V_{G}=-1 \mathrm{~V}$. The channel length modulation factor $\lambda$ is extracted from the linear fitting. (b) The dependence of $\lambda$ on the channel length $L$.

length from 2 to $50.5 \mu \mathrm{m}$. The dependence of $\lambda$ on channel length is plotted in Fig. 4(b). Look into Fig. 4(b), we can see the $\lambda$ is dramatically increasing when the channel length is below $5 \mu \mathrm{m}$, indicating an evident short channel effect. From this figure, the coefficient $\lambda$ is denoted as $0.83 / L$, where $L$ is in $\mu \mathrm{m}$.

\section{Extraction of subthreshold slope SS}

In subthreshold regime, the subthreshold slope $S S$ is defined as $S S=V_{G} / \log \left|I_{D}\right|$. The $S S$ is extracted from the transfer curves of the 50.5- $\mu \mathrm{m}$ channel length transistor, shown in Fig. 5. For this device, the $S S$ is as low as $0.17 \mathrm{~V} / \mathrm{dec}$, implying a fast transition between off state and on state in EGOFETs. In this model, the $S S$ is used to estimate the parameter $V_{S S}=S S / 2$ [28].

\section{E. Extraction of contact resistance $R_{C O}$}

To give the contact resistance referred above, the PTTTT transistors at six channel lengths from 2 to $50.5 \mu \mathrm{m}$ were presented here. The channel resistance of each device at $V_{G}=V_{D}=-1 \mathrm{~V}$ is graphed in Fig. 6(a) as the hollow squares. A linear fitting of these resistances indicates an $R_{C} \times W$ of 0.019 $\mathrm{M} \Omega \cdot \mathrm{cm}$ at $V_{G}=V_{D}=-1 \mathrm{~V}$, corresponding $R_{C 0}=0.12 \mathrm{M} \Omega$ at $W=1000 \mu \mathrm{m}$. As expected [14], the contact resistance in EGOFETs in much lower than that of $\mathrm{SiO}_{2}$-dielectric organic transistors [29]. 

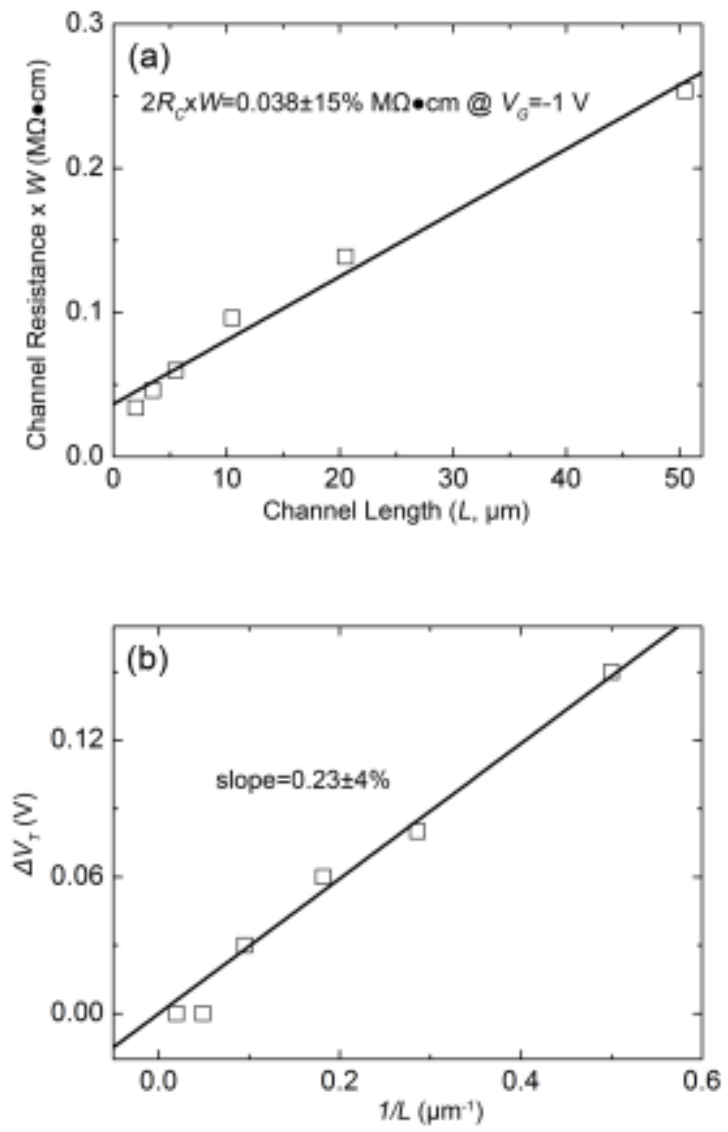

Fig. 6. (a) The channel resistance of EGOFETs at $V_{D}=V_{G}=-1 \mathrm{~V}$ with various channel lengths. (b) The threshold voltage $V_{T}$ shifts with the channel length $L$, caused by short channel effect.

\section{F. Extraction of $V_{T}$ shift coefficient $\zeta$ and $\delta_{V T}$}

Here, the threshold voltage of the $50.5-\mu \mathrm{m}$ channel length transistor is considered to the long channel threshold voltage $V_{T, L}$. The threshold voltage shift $V_{T, L}-V_{T}$ versus $1 / L$ is shown in Fig. 6(b). The slope of the linear fitting in Fig. 6(b) is $V_{T, L} \zeta$, so the threshold voltage shift coefficient $\zeta$ is obtained to be 0.77 . The magnitude of is $\delta_{\mathrm{vT}}$ usually very small [33], here we have $\delta_{V T}=0.1$, by linear fitting the threshold voltage extracted at different bias.

\section{G. Comparison between calculation and experimental data}

Fig. 7(a) shows the output characteristics $\left(I_{D^{-}} V_{D}\right)$ of the transistor with $L=50.5 \mu \mathrm{m}$ mentioned above. The drain voltage was swept from 0 to $-1 \mathrm{~V}$ with a $-0.02 \mathrm{~V}$ step, while the gate voltage was varied from 0 to $-1 \mathrm{~V}$ with a step of $-0.1 \mathrm{~V}$. The simulated curves given by our model match well with the symbolic circles from experimental data. The model parameters and their values in the simulation are summarized in Table I. The transistor parameters $W=1000 \mu \mathrm{m}$ and $L=50.5 \mu \mathrm{m}$ are given by the experimental conditions, while the parameters $V_{T}=-0.27 \mathrm{~V}, V_{s s}=0.085 \mathrm{~V}, R_{C 0}=0.12 \mathrm{M} \Omega, \gamma=1.33, \chi=0.67$, $\lambda=0.02, \quad \zeta=0.77$, and $\delta_{V T}=0.1$ are extracted from the experimental data, as discussed above. Only three parameters are obtained from fitting and they are $C_{0}=5 \mu \mathrm{F} / \mathrm{cm}^{2}, C_{v}=1.5$ $\mu \mathrm{F} / \mathrm{cm}^{2}$ at $\left|V_{G T}\right|=1 \mathrm{~V}$, and $\mu_{0}=0.028 \mathrm{~cm}^{2} / \mathrm{Vs}$. The ratio $C_{0} / C_{v}$ is
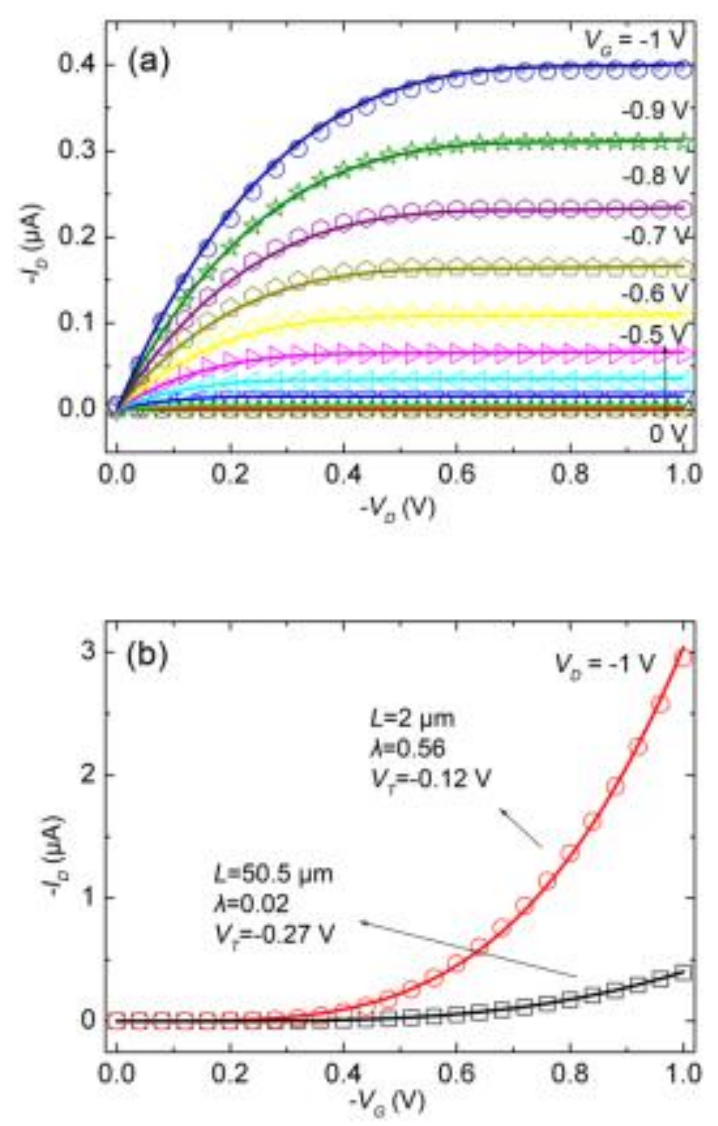

Fig. 7. (a) The comparison between calculation and experimental data for output characteristics of EGOFETs. (b) The comparison between calculation and experimental data for transfer characteristics of EGOFETs with $L=2$ and $50.5 \mu \mathrm{m}$. The symbolic circles are experimental, while the curves are simulated results given by the model.

around 3.3, which is comparable in the electric double layer capacitance modeled by two branches method [35]. Besides the magnificent low operation voltage, the capacitance is over 5 $\mu \mathrm{F} / \mathrm{cm}^{2}$, which is much higher than ordinary organic field effect transistors with gate dielectric. This implies a superior capability of polymer electrolyte as a gate dielectric. The transfer characteristics of the 50.5 and $2-\mu \mathrm{m}$ transistors are presented in Fig. 7(b) to show a further verification of our model. Their parameters share almost the same values except those listed in the figure. For both of them, we can find good agreements between experimental data (circles) and theoretical curves.

To investigate the validation of this model on different polymer semiconductor, sixty P3HT transistors at the same six channel length are presented here. The P3HT transistors shared the same fabrication processes with the PTTTT devices except different polymer semiconductors. In the same way, we extract the parameters $V_{T}=-0.2 \mathrm{~V}, R_{C 0}=0.06 \mathrm{M} \Omega, \lambda=0.6 / L$ for the P3HT transistors and the fitted mobility is given as $0.1 \mathrm{~cm}^{2} / \mathrm{Vs}$. By fixing all the other parameters but channel length, we plot the channel current dependence on the channel length for P3HT transistors in Fig. 8(a), where a good agreement we can see. The black squares with error bars are the currents from experimental data, which are matched well by the simulated 

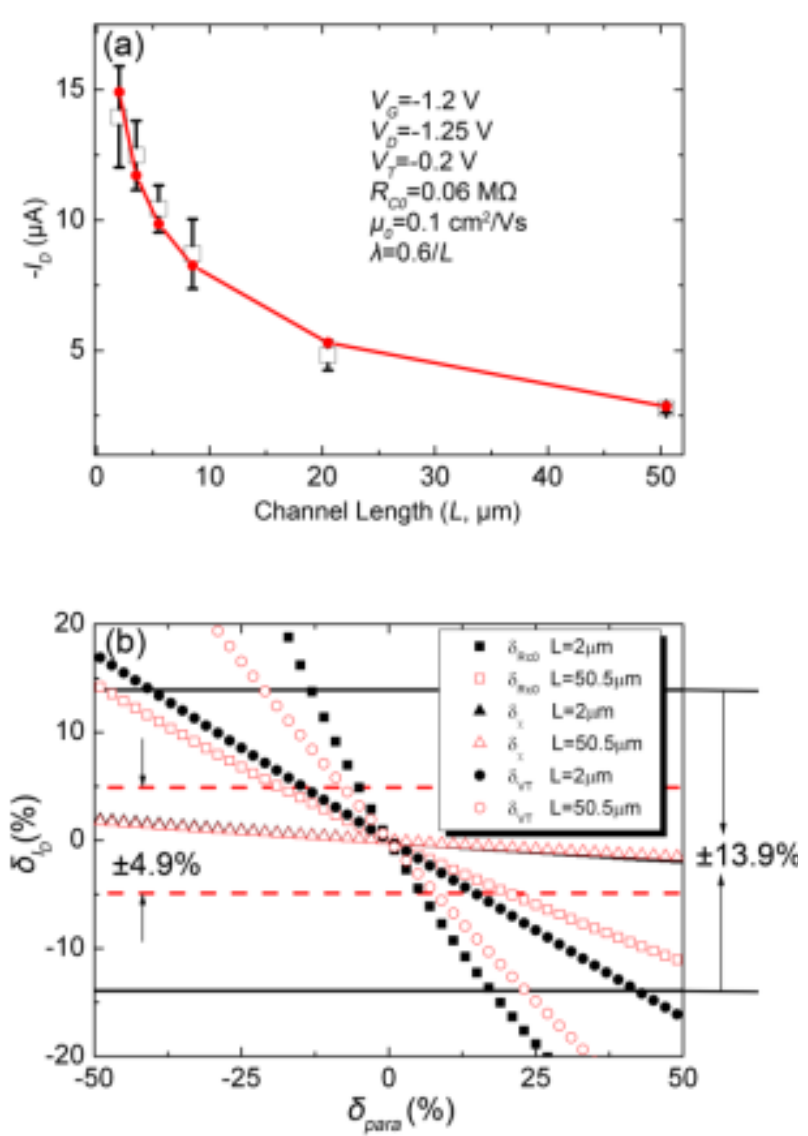

Fig. 8. (a) The channel currents at $V_{D}=-1.2 \mathrm{~V}$ and $V_{G}=-1.25 \mathrm{~V}$ of P3HT EGOFETs with various channel lengths. The circles are given by this model and the hollow squares are the mean value of measurement results. (b) The drain current variations caused by parameter variations.

current represented by red circles. This agreement indicates that the model is valid for devices fabricated in different batches.

We analyze the model sensitivity to parameter variation $\left(\delta_{\text {para }}\right)$, which leads to a drain current variation $\left(\delta_{I D}\right)$, and the results are presented in Fig. 8(b). Three parameters are analyzed here, including contact resistance $\left(R_{C O}\right)$, EDLC voltage dependent factor $(\chi)$, and threshold voltage $\left(V_{T}\right)$. From the statistic experimental data, the current of $2-\mu \mathrm{m}-L$ P3HT EGOFETs exhibits a variation of $\pm 13.9 \%$ (solid line in Fig. $8(\mathrm{~b}))$, which is higher than that of $50.5-\mu \mathrm{m}-L$ devices $( \pm 4.9 \%$, dash line in Fig. 8(b)). When $R_{C O}$ varies from $-13 \%$ to $17 \%$ or $-19 \%$ to $20 \%$, the model results stay in the interval of experimental data, for the P3HT EGOFETs with channel length of 2 or $50.5 \mu \mathrm{m}$, respectively. The long channel devices exhibit a better tolerance to contact resistance variation slightly. On the contrary, the long channel devices are quite sensitive to threshold voltage variation. The variation of $\pm 8 \%$ in $V_{T}$ leads a current variation of $\pm 4.9 \%$ for $50.5-\mu \mathrm{m}-L$ P3HT transistors, while the currents are agreed with experiment data even though $V_{T}$ varies from $-40 \%$ to $+42 \%$ for $2-\mu \mathrm{m}-L \mathrm{P} 3 \mathrm{HT}$ transistors. The current variation caused by variation of $\chi$ is less than $\pm 2 \%$ for all the devices in this case, when $\chi$ varies in $\pm 50 \%$. This is expected as the discussed voltage dependence of EDLC above.
TABLE I

MODEL PARAMETERS AND THEIR VALUES FOR 50.5- $\mu \mathrm{M}-L$ EGOFET

\begin{tabular}{llll}
\hline \hline \multicolumn{1}{c}{ Model parameters } & Notation & Unit & Value \\
\hline Type of transistor & $\mathrm{p}, \mathrm{n}$ & - & $\mathrm{p}$ \\
Channel width & $W$ & $\mu \mathrm{m}$ & 1000 \\
Channel length & $L$ & $\mu \mathrm{m}$ & 50.5 \\
Threshold voltage & $V_{T, L}$ & $\mathrm{~V}$ & -0.27 \\
Steepness of subthreshold regime & $V_{S S}$ & $\mathrm{~V}$ & 0.085 \\
Contact resistance & $R_{C O}$ & $\mathrm{M} \Omega$ & 0.12 \\
Mobility enhancement factor & $\gamma$ & - & 1.33 \\
EDLC factor & $\chi$ & - & 0.67 \\
Channel length modulation factor & $\lambda$ & $\mathrm{V}^{-1}$ & 0.02 \\
Threshold voltage shift factor & $\zeta$ & $\mu \mathrm{m}$ & 0.77 \\
Sensitivity of $V_{T}$ on bias & $\delta_{V T}$ & $\mathrm{~V}^{-1}$ & 0.1 \\
Voltage-independent capacitance & $C_{0}$ & $\mu \mathrm{F} / \mathrm{cm}^{2}$ & 5 \\
Voltage-dependent capacitance & $C_{v}$ & $\mu \mathrm{F} / \mathrm{cm}^{2} \mathrm{~V}$ & 1.5 \\
Low field mobility & $\mu_{0}$ & $\mathrm{~cm}^{2} / \mathrm{Vs}$ & 0.028
\end{tabular}

Although this model is proposed and verified for EGOFETs, it is quite general. For ordinary organic field effect transistors with gate dielectric, our model is still valid as long as we cut off the contribution from the electric double layer capacitance, by removing the part of voltage-dependent capacitance. This is quite reasonable if we consider the gate dielectric as a plate capacitor in absence of electric double layer.

\section{V.CONCLUSION}

In summary, we have developed a DC model for EGOFETs. This model is based on charge drift in presence of electric double layer capacitor. The voltage-dependent charge contribution of the electric double layer is considered and contact resistance is taken into account as well as short channel effect, which leads to non-saturation and threshold voltage shift. The model parameters and coefficients are extracted for the device simulation. Comparisons between experimental data and model theoretical simulations exhibit good agreements. The model is also applicable for conventional organic field effect transistors with insulating gate dielectrics.

\section{REFERENCES}

[1] H. Sirringhaus, N. Tessler and R. H. Friend, "Integrated optoelectronic devices based on conjugated polymers," Science vol. 280, no. 5370, pp. 1741-1744, Jun. 1998.

[2] M. Berggren, D. Nilsson, and N. D. Robinson, "Organic materials for printed electronics," Nature Mater., vol. 6, no. 1, pp. 3-5, Jan. 2007.

[3] F. Garnier, R. Hajlaoui, A. Yassar, and P. Srivastava, "All-polymer field-effect transistor realized by printing techniques," Science, vol. 265, no. 5179, pp. 1684-1686, Sep. 1994.

[4] Z. Bao, A. Dodabalapur, and A. J. Lovinger, "Soluble and processable regioregular poly(3-hexylthiophene) for thin film field-effect transistor applications with high mobility," Appl. Phys. Lett., vol. 69, no. 26, pp. 4108-4110, Dec. 1996.

[5] H. Sirringhaus, T. Kawase, R. H. Friend, T. Shimoda, M. Inbasekaran, W. $\mathrm{Wu}$, and E. P. Woo, "High-resolution inkjet printing of all-polymer transistor circuits," Science, vol. 290, no. 5499, pp. 2123-2126, Dec. 2000.

[6] J. Tardy, M. Erouel, A. Deman, A. Gagnaire, V. Teodorescu,M. Blanchin, B. Canut, A. Barau, and M. Zaharescu, "Organic thin film transistors with $\mathrm{HfO} 2$ high-k gate dielectric grown by anodic oxidation or deposited by sol-gel," Microelectron. Reliab., vol. 47, no. 2-3, pp. 372-377, Feb.-Mar. 2007. 
[7] C. Dimitrakopoulos, S. Purushothaman, J. Kymissis, A. Callegari, and J. M. Shaw, "Low-voltage organic transistors on plastic comprising high dielectric constant gate insulators," Science, vol. 283, no. 5403, pp. 822-824, Feb. 1999.

[8] M. Halik, H. Klauk, U. Zschieschang, G. Schmid, C. Dehm, M. Schutz, S. Maisch, F. Effenberger, M. Brunnbauer, and F. Stellacci, "Low-Voltage Organic Transistors with a Novel Molecular Gate Dielectric," Nature, vol. 431, no. 7011, pp. 963-966, Oct. 2004.

[9] A. S. Dhoot, J. D. Yuen, M. Heeney, I. McCulloch, D. Moses, and A. J. Heeger, "Beyond the metal-insulator transition in polymer electrolyte gated polymer field-effect transistors," Proc. Natl. Acad. Sci. USA, vol. 103, 11834-837, Aug. 2006.

[10] M. J. Panzer, C. R. Newman, C. D. Frisbie, "Low-voltage operation of a pentacene field-effect transistor with a polymer electrolyte gate dielectric," Appl. Phys. Lett., vol. 86, no. 10, pp. 103503, Feb. 2005

[11] D. Nilsson, N. Robinson, M. Berggren, and R. Forchheimer, "Electrochemical logic circuits," Adv. Mater., vol. 17, no. 3, pp. 353-358, Feb. 2005.

[12] D. A. Bernards and G. G. Malliaras, "Steady-state and transient behavior of organic electrochemical transistors," Adv. Funct. Mater., vol. 17, no. 17, pp. 3538-3544, Oct. 2007.

[13] L. Herlogsson, X. Crispin, N. D. Robinson, M. Sandberg, O. -J. Hagel, G. Gustafsson, and M. Berggren, "Low-voltage polymer field-effect transistors gated via a proton conductor," Adv. Mater., vol. 19, no. 1, pp. 97-01, Jan. 2007.

[14] L. Herlogsson, Y. -Y. Noh, N. Zhao, X. Crispin, H. Sirringhaus, and M. Berggren, "Downscaling of organic field-Effect transistors with a polyelectrolyte gate insulator," Adv. Mater., vol. 20, no. 24, pp. 4708-4713, Dec. 2008

[15] L. Herlogsson, M. Cölle, S. Tierney, X. Crispin, and M. Berggren, "Low-voltage ring oscillators based on polyelectrolyte-gated polymer thin-film transistors," Adv. Mater., vol. 22, no. 1, pp. 72-76, Jan. 2010.

[16] J. R. Miller and P. Simon, "Electrochemical capacitors for energy management," Science, vol. 321, no. 5889, pp. 651-652, Aug. 2008

[17] S. Mitra, A. K. Shukla, and S. Sampath, "Electrochemical capacitors with plasticized gel-polymer electrolytes," J. Power Source, vol. 101, no. 2, pp. 213-218, Aug. 2001.

[18] G. D. Wilk, R. M. Wallace, and J. M. Anthony, "High-к gate dielectrics: Current status and materials properties considerations," J. Appl. Phys., vol. 89, no. 10, pp. 5243 -5275, May 2001.

[19] M. Jacunski, M. Shur, A. Owsu, T. Ytterdal, M. Hack, and B. Iniguez, "A short-channel DC SPICE model for polysilicon thin-film transistors including temperature effects," IEEE Trans. Electron Devices, vol. 46, no. 6, pp. 1146-1158, Jun. 1999

[20] M. Shur and M. Hack, "Physics of amorphous silicon based alloy field-effect transistors," J. Appl. Phys., vol. 55, no. 10, pp. 3831-3842, May 1984

[21] M. Vissenberg and M. Matters, "Theory of the field-effect mobility in amorphous organic transistors," Phys. Rev. B, vol. 57, no. 20, pp. 12964-12967, May 1998.

[22] B. E. Conway, Electrochemical supercapacitors - Scientific fundamentals and technological applications, Kluwer Academic/Plenum Publishers, New York, 1999.

[23] J. J. Lyklema, Fundamentals of Interface and Colloid Science, Academic Press, 1995, vol. 2, pp. 208.

[24] S. Lamperski and C. W. Outhwaite, "Differential capacitance of the solvent primitive model diffuse layer using the inhomogeneous Poisson-Boltzmann theory with an exclusion volume term," Journal of Electroanalytical Chemistry, vol. 567, no. 2, pp. 263-267, Jun. 2004.

[25] V. V. Emets, B. B. Damaskin, and I. A. Bagotskaya, "Electrical Double Layer Structure in Surface-Inactive Electrolytes on a Liquid $\mathrm{Cd}-\mathrm{Ga}$ Electrode in Methanol and Propylene Carbonate," Russian Journal of Electrochemistry, vol. 37, no. 1, pp. 88-94, Jan. 2001.

[26] S. Locci, M. Morana, E. Orgiu, A. Bonfiglio, and P. Lugli, "Modeling of short-channel effects in organic thin-film transistors," IEEE Trans. Electron Devices, vol. 55, no. 10, pp. 2561-2567, Oct. 2008.

[27] L. Li, H. Marien, J. Genoe, M. Steyaert, and P. Heremans, "Compact model for organic thin-film transistor," IEEE Electron Device Lett., vol. 31, no. 3, pp. 210-212, Mar. 2010.

[28] O. Marinov, M. J. Deen, U. Zschieschang, and H. Klauk, "Organic thin-film transistors: part I-compact DC modeling," IEEE Trans. Electron Devices, vol. 56, no. 12, pp. 2952-2961, Dec. 2009.

[29] H. Klauk, G. Schmid, W. Radlik, W. Weber, L. Zhou, C. D. Sheraw, J. A. Nichols and T. N. Jackson, "Contact resistance in organic thin film transistors," Solid State Electron., vol. 47, pp 297-301, Feb. 2003.
[30] D. Natali, L. Fumagalli and M. Sampietro, "Modeling of organic thin film transistors: Effect of contact resistances," J. Appl. Phys., vol. 101, no. 1, pp. 014501, Feb. 2007.

[31] J. N. Haddock, X. Zhang, S. Zheng, Q. Zhang, S. R. Marder, and B. Kippelen, "A comprehensive study of short channel effects in organic field-effect transistors," Org. ELectron., vol. 7, no. 1, pp. 45-54, Jan. 2006.

[32] W. Fichtner and H. W. Pötzl, "MOS Modelling by Analytical Approximations," Int. J. Electron., vol. 46, no. 1, pp. 33-55, Jan. 1979

[33] M. J. Deen, O. Marinov, U. Zschieschang, and H. Klauk, "Organic Thin-Film Transistors: Part II-Parameter Extraction," IEEE Trans. Electron Devices, vol. 56, no. 12, pp. 2962-2968, Dec. 2009.

[34] G. J. Hu, C. Chang, and Y. -T. Chia, "Gate-voltage-dependent effective channel length and series resistance of LDD MOSFET's," IEEE Trans. Electron Devices, vol. 34, no. 12, pp. 2469-2475, Dec. 1987.

[35] F. Belhachemi, and S. Rael and B. Davat, "A physical based model of power electric double-layer supercapacitors," IEEE-IAS'00, pp.3069-3076, Rome, Italy, Oct. 2000.

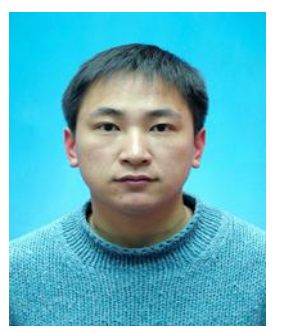

Deyu Tu received the B.S. degree in mechanism engineering and automatization from the Tsinghua University, Beijing, China, in 2003 and the Ph.D. degree in microelectronics and solid-state electronics from the Institute of Microelectronics, Chinese Academy of Sciences, Beijing, in 2008.

From 2008 to 2010, he was a Postdoctoral Researcher at National Nanotechnology Laboratory of Consiglio Nazionale delle Ricerche-Istituto Nanoscienze, Lecce, Italy. He joined the Information Coding of Linköping University for the "OPEN" project in 2010. His research activities focus on the modeling and simulation of organic electronic devices and circuits, as well as the fabrication and characteristics.

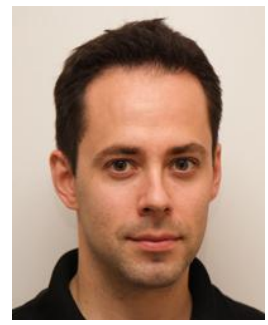

Lars Herlogsson recieved the M.Sc. degree in applied physics and electrical engineering from Linköping University, Sweden, in 2001.

From 2001 to 2004, he was a research engineer at Thin Film Electronics AB, Linköping, Sweden, where he worked with manufacturing, characterization and development of non-volatile memories based on ferroelectric polymers. In 2005, he joined the Organic Electronics at Linköping University, where he is currently pursuing the Ph.D. degree. His research focuses on electrolyte-gated organic thin-film transistors for low-voltage electronics.

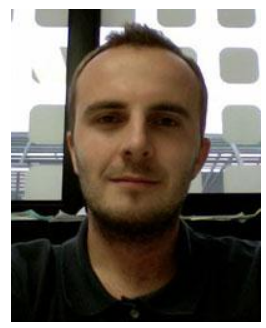

Loïg Kergoat received his M. S. degree in physical chemistry from the University of Paris Diderot, Paris, France, in 2007, during which he spent 4 months at the Van't Hoff Institute for Molecular Sciences, University of Amsterdam, Amsterdam, Netherlands, and the $\mathrm{PhD}$ degree in physical chemistry from the University of Paris Diderot in 2010, with a thesis about organic transistors and their use in biosensing applications.

$\mathrm{He}$ is currently a postdoctoral researcher at the Department of Science and Technology (ITN), Linköping University, Norrköping, Sweden. His research field concerns electrolyte-gated organic thin-film transistors.

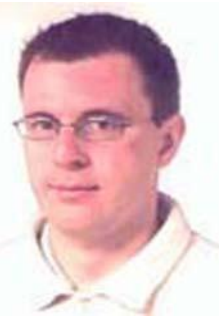

Xavier Crispin graduated from the University of Mons-Hainaut in 1995, in Chemistry. From 1995 to 2000 he was graduate student of Prof. J.L. Brédas in the Laboratory of the Chemistry of Novel Materials and Research Center in Molecular Electronics and Photonics.

In 2000, he received an individual Marcie-Curie Fellowship and spent two years as postdoctoral fellow at Linköping University with Prof. W. R. Salaneck. He has been trained to become a spectroscopist using various techniques (XPS, ARUPS, NEXAFS) to characterize the electronic structure of conjugated materials, the way they order and their interfaces with electrodes. He was a member of the Laboratory of Surface Physics and Chemistry, Department of Physics, Chemistry and Biology, 
Linköping University until 2004. In 2003, he acquires docent competence at Linköping universitet and teaches in the areas of molecular physics and organic electronics. Since 2004, Xavier is Associate Professor in the group of Prof. M. Berggren in Norrköping Campus, Linköping University.

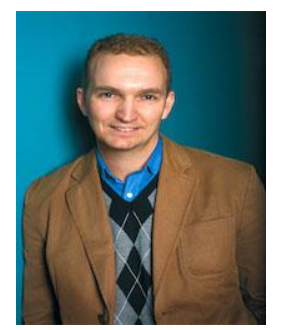

Magnus Berggren received his MSc in Physics from Linköping University in 1991 and completed his $\mathrm{PhD}$ at the Laboratory of Applied Physics, also at Linköpings Unviversitet, in 1996.

He then joined Bell Laboratories in Murray Hill, $\mathrm{NJ}$ in the USA, for a one-year post doc research period focusing on developing lasers which included organic electroactive materials as the lasing and gain medium. In 1997 he teamed up with Opticom ASA, in Norway, and former colleagues of Linköping University, as the managing director, to establish and build up Thin Film Electronics $\mathrm{AB}$, whose main business is to develop electronic memory devices based on ferroelectric polymers. After this, he returned to Linköping University and also to a part time position at Acreo AB. There, in 1999, he initiated several paper electronics projects supported in part by several paper- and packaging companies. Since 2001, he has been the professor in Organic Electronics at Linköping University and leads a research group of about 15 scientists. Also, since 2005 he has been the director of the Strategic Research Centre for Organic Bioelectronics.

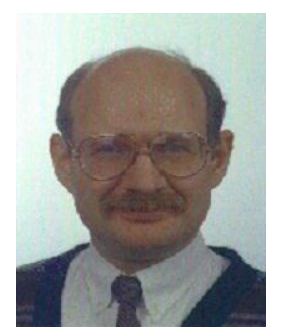

Robert Forchheimer was born in Gothenburg, Sweden in 1948. He received his Diploma degree in electrical engineering at the Royal Institute of Technology (KTH), Stockholm, Sweden in 1972 and Ph. D. in information theory at Linköping University, Linköping, Sweden in 1979.

He has held positions as Researcher and Lecturer at Linköping University, Visiting Scientist at Univ. of Southern California, USA and Visiting Professor at Universität Hannover, Germany. He is currently Professor at Linköping University where he heads a research group in Information Coding. His current interests are organic electronics, systems biology and optical networks. He has previously contributed to the fields of image and video coding, packet radio communication, smart optical sensors and optical computing.

Prof. Forchheimer was associate editor for IEEE Tr. Image Processing during 1996-1998. He has further been a member of Incentive Advisory Board and a member of the evaluation committee of the Swedish Board for Technical Development (NUTEK). In 2006 he became recipient of the Picture Coding Symposium (PCS) Award on the occasion of its 25th anniversary in recognition for his work in the picture coding field. 